\title{
Determination of Gamma Angular Distribution from the Shape of Spectral Line for the First Excited State of Carbon Nucleus
}

\author{
K. Rusiecka1 ${ }^{*}$, A. Wrońska1 ${ }^{*}$, P. Bednarczyk², D. Böckenhoff ${ }^{3}$, A. Bubak ${ }^{4}$, S. Feyen ${ }^{3}$,

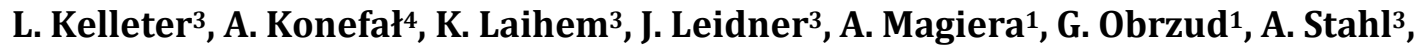 \\ M. Ziębliński² \\ ${ }^{1}$ Marian Smoluchowski Institute of Physics, Jagiellonian University, Kraków, Poland \\ ${ }^{2}$ Henryk Niewodniczański Institute of Nuclear Physics PAN, Kraków, Poland \\ ${ }^{3}$ RWTH Aachen University, Aachen, Germany \\ ${ }^{4}$ Department of Nuclear Physics and Its Applications, Institute of Physics, University of Silesia, Katowice, Poland \\ Email: "Katarzyna.Rusiecka@student.uj.edu.pl, *Aleksandra.Wronska@uj.edu.pl
}

Received 27 November 2015; accepted 8 January 2016; published 11 January 2016

Copyright (C) 2016 by authors and Scientific Research Publishing Inc.

This work is licensed under the Creative Commons Attribution International License (CC BY). http://creativecommons.org/licenses/by/4.0/

(c) (i) Open Access

\begin{abstract}
An experiment investigating gamma emission in hadron therapy was performed at Cyclotron Centre Bronowice (CCB), Cracow, Poland, using two different phantom materials-carbon and poly(methyl methacrylate) PMMA. The measurements were carried out at $70 \mathrm{MeV}$ proton beam energy and the gamma quanta were registered with the use of HP Ge detector with scintillation anti-Compton shielding. Although the primary aim was to establish a solid experimental data base for future applications in prompt gamma imaging, the data have also been analyzed with regards to the position and shape of the spectral line stemming from deexcitation of the carbon excited state 4.44 $\mathrm{MeV}$. Measurements potentially useful to determine the cross section were performed only at $90^{\circ}$ laboratory polar angle. However, benefiting from the very good energy resolution it turned out possible to extract information on angular distribution of the $C^{*}(4.44 \mathrm{MeV})$ deexcitation by analyzing the associated line shape. This paper presents the scheme of model calculations assuming the whole process can be divided into two stages: excitation of carbon nuclei by impinging protons and deexcitation of the $\mathrm{C}^{*}(4.44 \mathrm{MeV})$ state.
\end{abstract}

\section{Keywords}

Prompt Gamma Radiation, Proton Range Monitoring, Proton Therapy, Radiotherapy, Angular Distribution

\footnotetext{
${ }^{*}$ Corresponding authors.
}

How to cite this paper: Rusiecka, K., et al. (2016) Determination of Gamma Angular Distribution from the Shape of Spectral Line for the First Excited State of Carbon Nucleus. World Journal of Nuclear Science and Technology, 6, 63-70. 


\section{Introduction}

Proton beam therapy is a high-quality radiation therapy which uses proton beam to irradiate neoplastic tissue. The main advantage of this type of radiotherapy is highly conformal dose deposition due to the presence of the Bragg peak. It allows depositing a maximum dose in the area of abnormal tissue while healthy tissues receive only a marginal dose [1]. However, if the range of protons in the patient's body is not accurately known, then there is a risk of receiving an overdose by the organs at risk (OAR) or receiving an underdose by the treated volume (TV). To take a full advantage of the beneficial physical properties of the proton beam, it is often required to irradiate the tumor volume with a precision better than $1-2 \mathrm{~mm}$, which means that proton therapy needs not only precise treatment planning but also monitoring and proton range verification during the treatment [2]. Monitoring of the proton range can be performed by detecting gamma rays produced in two kinds of nuclear reactions occurring in the patient's body during the treatment: the production of positron-emitting isotopes and the excitation of the target nuclei by incident protons [1].

Positron emission tomography (PET) is considered to be a feasible method of monitoring and verification the dose delivered during the proton therapy and currently it is the only technique of particle therapy monitoring in clinical application [3]. However, there are several limitations to this method, including the fact that energy deposition during proton therapy mismatches the production of positron-emitting isotopes and the appearance of biological washout effects related to blood perfusion and metabolism. Furthermore, it has been observed that delay between treatment and taking PET scan affects determination of the range due to different half-lives of isotopes [2]. This issue can be overcome by real-time PET monitoring procedure, which is currently a subject of studies [4]. Nevertheless, comparison of PET images and Monte Carlo simulations can reveal the range more accurately [2].

Due to numerous drawbacks of PET dose monitoring, new and more effective solutions have been searched for. The approach based on the measurement of prompt gamma radiation seems to be among the most promising options. Prompt gamma radiation is produced in various interactions of a proton beam with the target nuclei e.g. inelastic scattering and excitation. Its emission takes place within less than one nanosecond after nuclear reaction and indicates the place of nuclear interactions; therefore it allows monitoring beam range in real time [3]. Gamma quanta created from deexcitation of excited states of ${ }^{12} \mathrm{C}$ and ${ }^{16} \mathrm{O}$ carry energy of several $\mathrm{MeV}$. As they interact rather weakly with human tissue, they bear almost undisturbed information.

The work presented in this article is part of the project Investigation of gamma emission in experimental modeling of hadron therapy, which aims to examine correlation between the characteristics of the gamma radiation emitted during proton therapy and the Bragg peak position. Research conducted within the project includes experiments designed to simulate course of the proton therapy as well as Monte Carlo simulations.

This paper presents analysis of experimental data with regards to the position and shape of the carbon line stemming from deexcitation of the first excited state of carbon nuclei. Mathematical model describing the shape of the carbon spectral line has been developed in order to reproduce experimental data, which allowed reconstructing angular distribution of the nuclear process occurring in patient's body during treatment.

\section{Experimental Technique}

A series of measurements were carried out in Cyclotron Centre Bronowice, Cracow, Poland in order to obtain data on gamma-emission from the interaction of proton beam with phantoms of the elemental composition simulating human tissue. The experimental conditions were designed to deliver data useful for hadron radiotherapy. The proton beam with energy of $70 \mathrm{MeV}$ was accelerated in the Proteus C-235 cyclotron. Figure 1 shows schematically the experimental setup. Intensity of the beam was controlled using a Beam Current Monitor (BCM) (1) in form of a pair of telescopes made of plastic scintillators. The telescopes registered protons scattered (2) on the titanium exit window of the beam pipe (3) with the rate proportional to the intensity of the beam. The data registered by BCM was used for relative normalization of obtained gamma spectra.

The phantom with adjustable thickness (thick phantom) (4) and an additional thin target of the same material and fixed thickness (5) were placed on the beam path (6). Two materials of phantoms were investigated so far: poly(methyl methacrylate) PMMA $\left(\mathrm{C}_{5} \mathrm{O}_{2} \mathrm{H}_{8}\right)_{\mathrm{n}}$ with density of $1.19 \mathrm{~g} \cdot \mathrm{cm}^{-3}$ and graphite with density of $1.74 \mathrm{~g} \cdot \mathrm{cm}^{-3}$.

The gamma rays were detected using two HP Ge detectors with anti-Compton shielding (7). The detectors were mounted on movable platforms, which allowed to register gamma spectra at different angles for different 


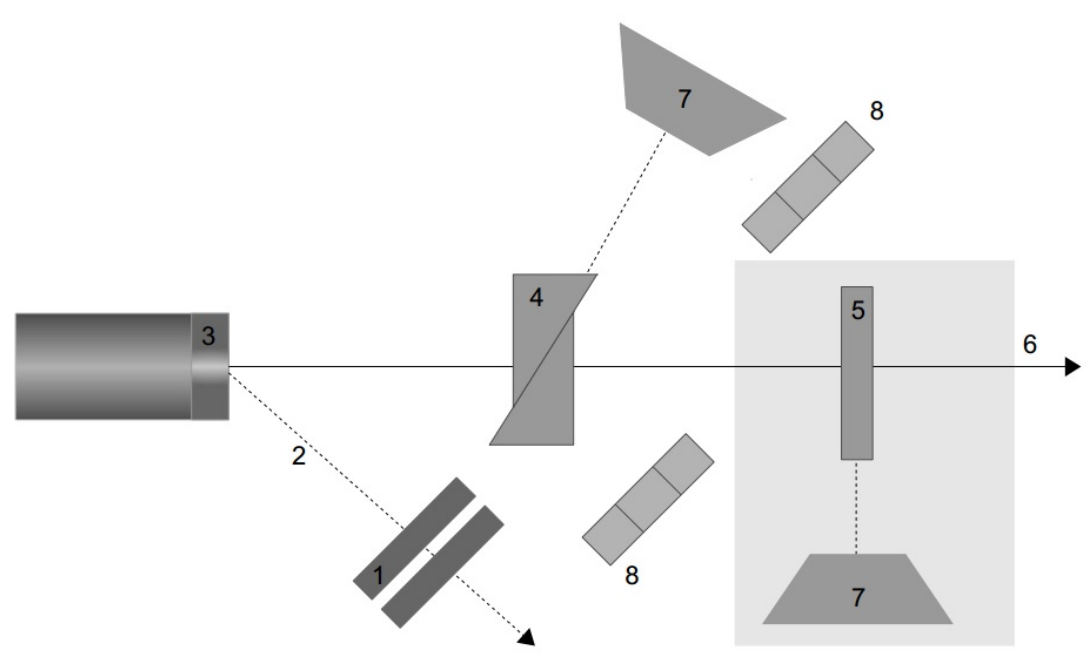

Figure 1. Scheme of experimental setup (not to scale). 1-beam current monitor, 2scattered protons, 3-window of the beam pipe, 4 - thick target (variable thickness), 5-thin target (fixed thickness), 6-beam path, 7-HP Ge detectors with anti-Compton shielding, 8-lead shielding. Data presented in the article were registered from the highlighted part of the setup.

measurement series. Each of the detectors was collecting data from different parts of the experimental setup: first one from the thick phantom, and second one from the thin target. This configuration of detection system enables an integrated gamma emission study along with a differential measurement. Additionally, lead walls (8) were placed as shown in Figure 1, in order to shield detectors from radiation emitted from the other target (the one that the detector was not directed onto).

The parameters which were fixed during a single measurement series were the following: proton beam energy, detection angle and phantom material, while the variable parameter was the phantom thickness. Change of the thick phantom thickness during a measurement series allows modifying average proton energy inside the thin target in small, precise steps.

Experimental data presented in this paper were obtained in measurements with graphite targets and a HP Ge detector placed at $90^{\circ}$ to the beam direction, collecting data from the thin target. Thick target thickness has been changed from $15 \mathrm{~mm}$ up to $30 \mathrm{~mm}$ which resulted in average proton energy inside the thin target changing from 41.16 MeV till complete stopping of proton beam. Average energy was estimated with Geant4 simulation, as the mean value of the energy distribution for those protons passing the thin target, which were still able to excite the 4.44 MeV carbon state.

\section{Shape of the ${ }^{12} \mathrm{C}_{4.44 \rightarrow \text { g.s. }}$ Line-The Model}

The ${ }^{12} \mathbf{C}_{4.44 \rightarrow \text { g.s. }}$ line is visible in the obtained gamma spectra as a double peak (Figure 2). The explanation of its shape lies in the interplay of the Doppler shift and the selective population of magnetic sublevels of the first excited state of carbon nuclei in the inelastic scattering process [5]. This phenomenon has been also observed in the gamma spectra registered from solar flares and molecular clouds [6].

The interaction between target nuclei and incident protons occurs in two stages (Figure 3). In the first step incident proton causes excitation of the target nucleus [5]. Angular distribution in the centre-of-mass for this process is typically presented in the basis of Legendre polynomials $P_{l}$ as follows:

$$
W_{1}(\theta)=\sum_{l=0}^{l=l_{\max }} c_{l} P_{l}\left(\cos \theta_{C M}\right)(2 l+1)
$$

The coefficients $c_{l}$ have been calculated for each studied proton energy using the TALYS package [7].

In the second step, deexcitation of the excited nucleus occurs along with the emission of the gamma quantum. Angular distribution of this process in the rest frame of the excited carbon nucleus is given by: 


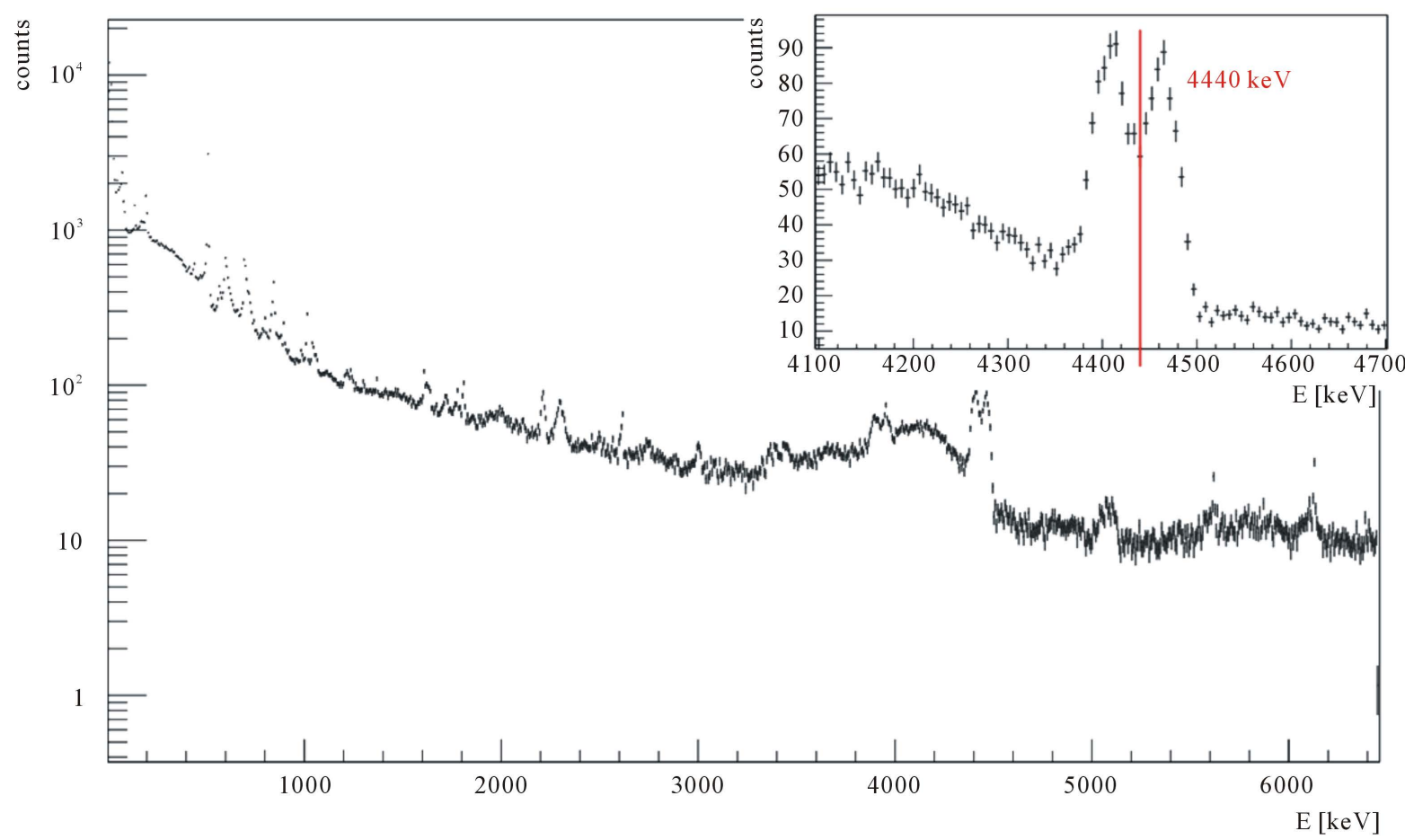

Figure 2. Typical gamma spectrum registered from the thin target in described experimental conditions. Average energy of protons inside the thin target was $16.13 \mathrm{MeV}$.

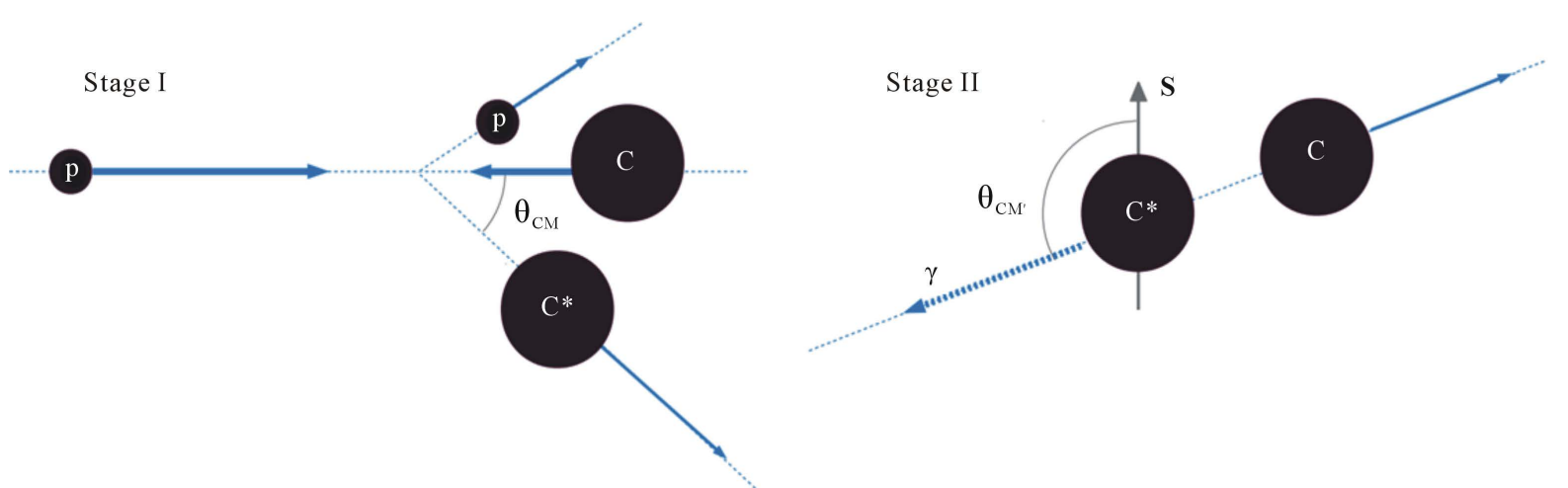

Figure 3. The scheme of two-step reaction of protons interacting with matter. Step I: excitation of ${ }^{12} \mathrm{C}$ nucleus, step II: deexcitation of the excited state with emission of gamma quantum.

$$
W_{2}\left(\theta_{C M^{\prime}}\right)=\sum_{m=-2}^{m=2} a_{m}\left|Y_{2}^{m}\left(\theta_{C M^{\prime}}, \phi_{C M^{\prime}}\right)\right|^{2},
$$

where $a_{m}$ is probability for populating the $m$ sublevel and $Y_{L}^{m}$ are spherical harmonics, where the choice of $L=2$ is given by the difference of spins of excited and ground states. In the performed calculation, as a quantization axis a normal to the scattering plane has been chosen [5]. Coefficients $a_{m}$ are unknown and they are calculated based on the experimental data. Due to holding following constraints:

$$
\begin{aligned}
& a_{m}=a_{-m}, \\
& \sum a_{m}=1
\end{aligned}
$$

it is sufficient to determine only two of them (e.g. $a_{0}$ and $a_{1}$ ) to fully describe angular dependence of the ${ }^{12} \mathrm{C}_{4.44 \rightarrow \text { g.s. }}$ transition [6]. 


\section{Results}

Model calculations were carried out using ROOT data analysis framework [8]. For both reaction stages the products are generated uniformly in the respective phase spaces and the corresponding probabilities $W_{1}$ and $W_{2}$ are calculated based on the angles and assumed values of parameters $a_{0}$ and $a_{1}$. Subsequently, the product of those is used as a weight for each event. Model calculations account also for the finite size of the detector and its position as well as for the energy resolution of the detector estimated independently as FWHM of oxygen 6.13 MeV line, visible in the spectra obtained in measurements with a PMMA phantom.

Values of $a_{0}$ and $a_{1}$ coefficients have been determined by comparing experimental data on the ${ }^{12} \mathrm{C}_{4.44 \rightarrow \text { g.s. }}$ line shape with theoretical model and constructing map of $\chi^{2}$ distribution for all possible combinations of searched parameters. For a certain choice of parameters $a_{0}$ and $a_{1}$, a theoretical line shape is determined, and a $\chi^{2}$ for that and the experimental data is calculated as follows:

$$
\chi^{2}=\sum_{n} \frac{\left(X_{\text {exp }}-X_{\text {theor }}\right)^{2}}{\left(d X_{\text {exp }}\right)^{2}+\left(d X_{\text {theor }}\right)^{2}},
$$

where, $X_{\text {exp }}$ is experimental value, $X_{\text {theor }}$ is theoretical value calculated based on the model, $d X_{\text {exp }}$ and $d X_{\text {theor }}$ are respective uncertainties of those. The sum is calculated for $n$ bins in the studied energy interval. Typical $\chi^{2}$ distribution is shown in Figure 4. The triangle indicates area, where $a_{0}$ and $a_{1}$ coefficients have physically valid values, which is a consequence of (4). However, maps were constructed in a wider range to enhance the visibility of the minimum in those distributions and make determination of its position more precise. Values of $a_{0}$ and $a_{1}$ parameters are found as coordinates of the minimum of the $\chi^{2}$ distribution map. The cross on the $\chi^{2}$ distribution map (Figure 4) indicates the determined position of the minimum. Such $\chi^{2}$ distribution maps were calculated for each studied energy of protons inside the thin target, which allowed to determine values of $a_{0}$ and $a_{1}$ parameters for them. Values of $a_{0}$ and $a_{1}$ parameters determined for the analysed data series are shown in Figure 5 as a function of protons energy.

Figure 6 sets together theoretical line shape (calculated according to described model and with values of $a_{0}$ and $a_{1}$ determined as above) and the experimental data. Additionally, known parameters $a_{0}$ and $a_{1}$ fix the shape of the angular distribution of the reaction (Figure 7).

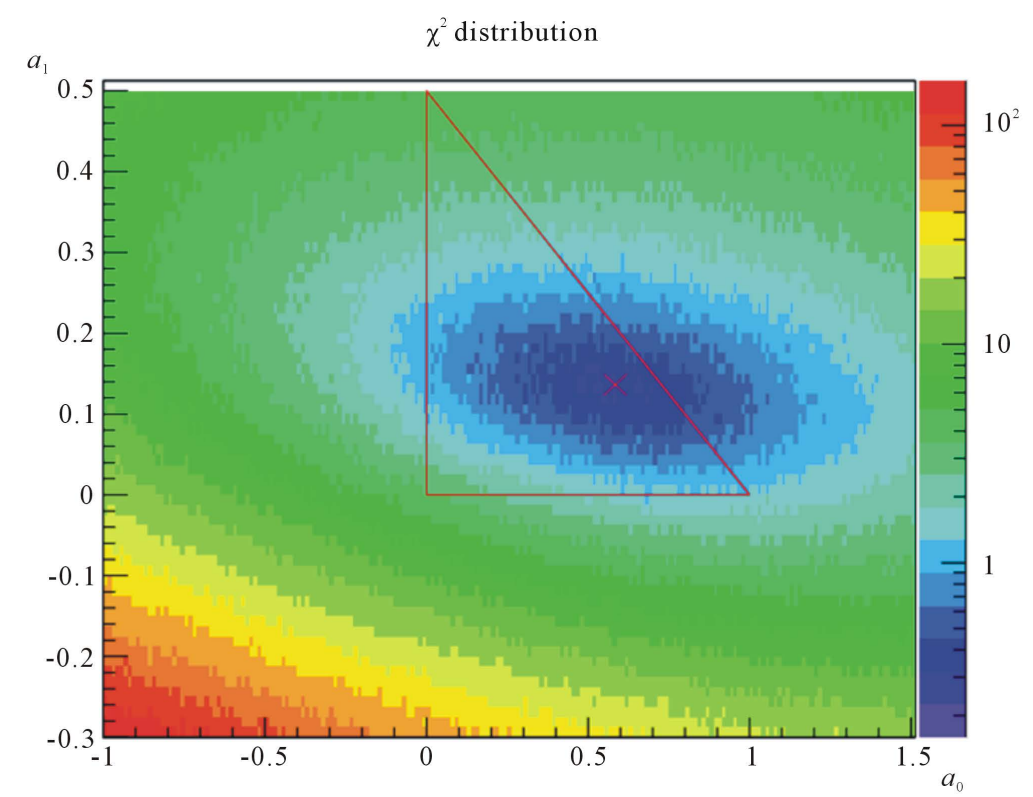

Figure 4. Typical map of $\chi^{2}$ distribution. The triangular shape indicates area, where $a_{0}$ and $a_{1}$ coefficients have physically valid values and red cross indicates the position of the minimum. Map has been constructed for mean proton energy in the target of $16.13 \mathrm{MeV}$ (preliminary results). 
Values of $a_{0}$ and $a_{1}$ parameters

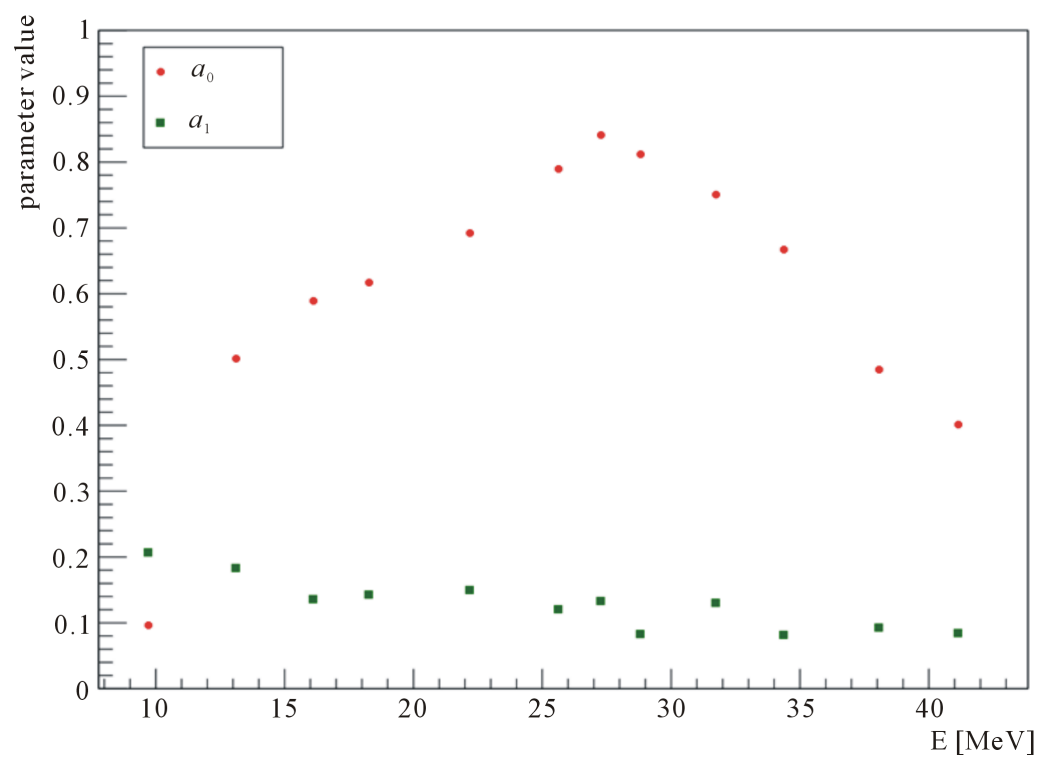

Figure 5. Values of $a_{0}$ and $a_{1}$ parameters as a function of protons energy (preliminary results).

${ }^{12} \mathrm{C} 4.44 \mathrm{MeV}$ - experimental and theoretical line

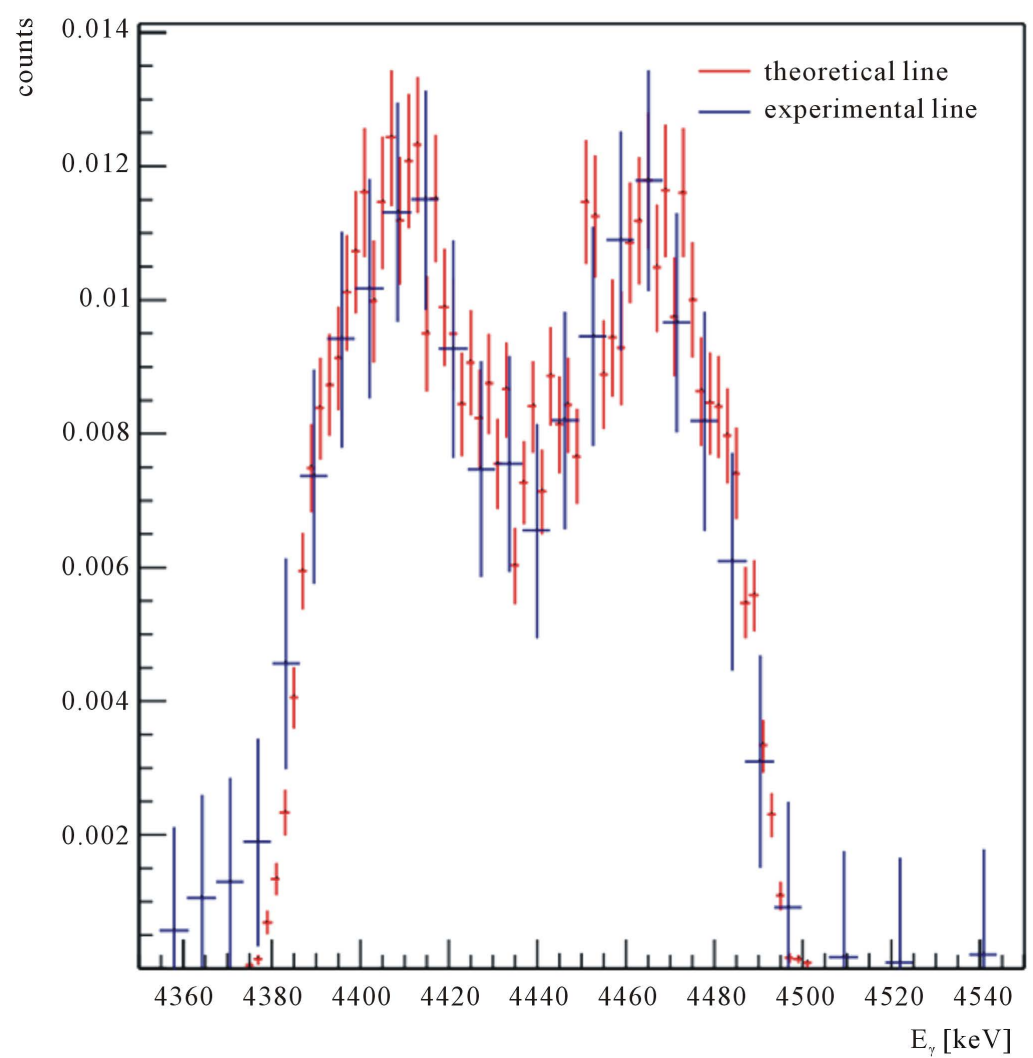

Figure 6. Comparison of the shape of experimental ${ }^{12} \mathrm{C}_{4.44 \rightarrow \text { a.s. }}$ line and theoretical line reconstructed based on mathematical model (preliminary results). Presented errors are purely statistical. 


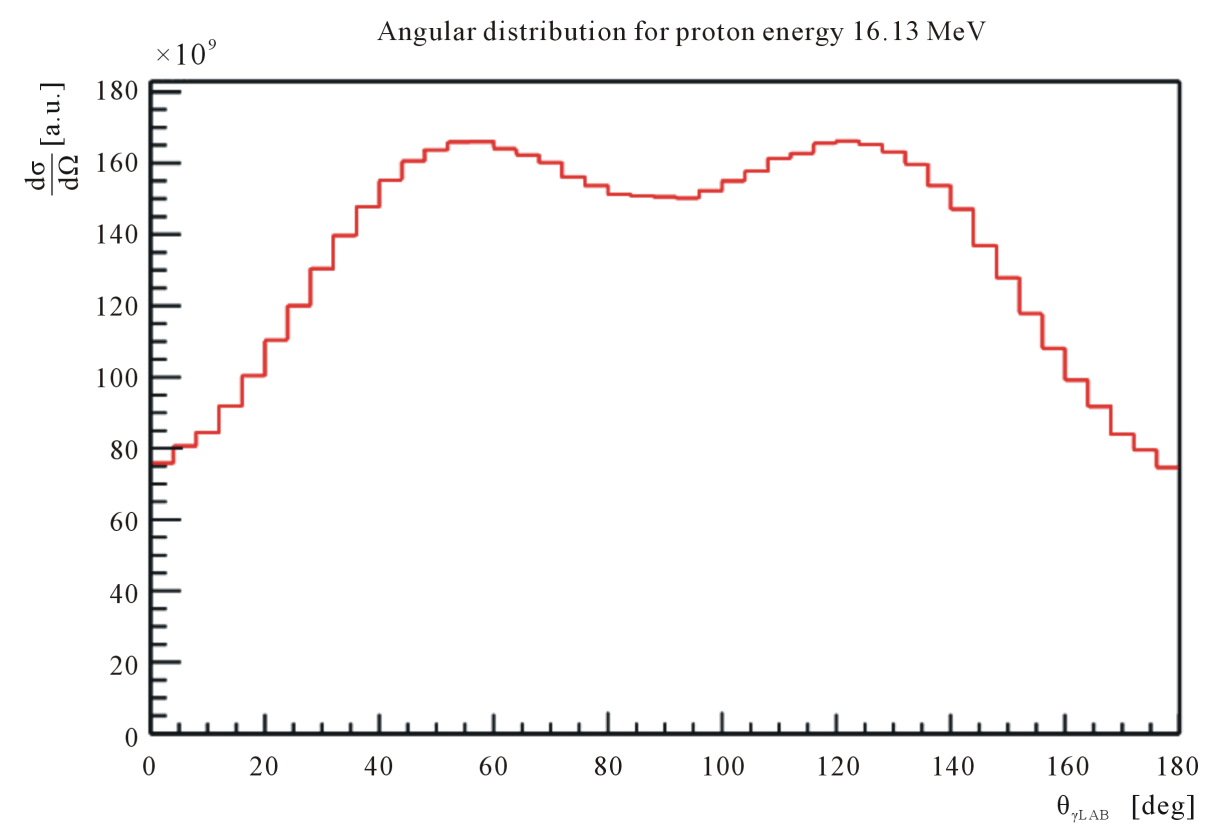

Figure 7. Angular distribution (not normalized absolutely) of gamma emission in ${ }^{12} \mathrm{C}_{4.44 \rightarrow \text { q.s. }}$, as determined based on $a_{0}$ and $a_{1}$ parameters calculated for proton energy of $16.13 \mathrm{MeV}$ (preliminary results).

\section{Summary and Further Research}

Mathematical model describing the shape of the ${ }^{12} \mathrm{C}_{4.44 \rightarrow \text { g.s. }}$ transition line has been developed in order to reproduce experimental data obtained in the measurements simulating the course of the proton therapy. As a result of model calculations, the values of parameters $a_{0}$ and $a_{1}$ were obtained. Those allow to fully reconstruct the shape of the carbon line as well as determine the angular distribution of the $C^{*}(4.44 \mathrm{MeV})$ deexcitation-a nuclear reaction occurring during proton therapy in patient's body. The elaborated method allows extracting full angular distribution of the process merely from the shape of the spectral line measured at a single detection angle. Further plans comprise measurements for beam energy of $150 \mathrm{MeV}$ and $230 \mathrm{MeV}$, which will cover full range of energy used in proton therapy, and also at various polar angles. The latter will enable a comparison of the model calculations with classical measurement of the angular distribution and thus will provide a rigorous test of the model.

\section{Acknowledgements}

Research was conducted in collaboration with the Institute of Nuclear Physics of the Polish Academy of Sciences and RWTH Aachen University.

The project Investigation of gamma emission in experimental modeling of hadron therapy is carried out within the POMOST programme of the Foundation for Polish Science, co-financed from the European Union under the European Regional Development Fund.

\section{References}

[1] Min, C.H., Kim, C.H., Youn, M.Y. and Kim, J.W. (2006) Prompt Gamma Measurements for Locating the Dose Falloff Region in the Proton Therapy. Applied Physics Letters, 89, Article ID: 183517. http://dx.doi.org/10.1063/1.2378561

[2] Moteabbed, M., España, S. and Paganetti, H. (2011) Monte Carlo Patient Study on the Comparison of Prompt Gamma and PET Imaging for Range Verification in Proton Therapy. Physics in Medicine and Biology, 56, 1063-1082. http://dx.doi.org/10.1088/0031-9155/56/4/012

[3] Pinto, M., et al. (2015) Absolute Prompt-Gamma Yield Measurements for Ion Beam Therapy Monitoring. Physics in Medicine and Biology, 60, 565-594. http://dx.doi.org/10.1088/0031-9155/60/2/565

[4] Kraan, A.C., et al. (2015) Online Monitoring for Proton Therapy: A Real-Time Procedure Using a Planar PET System. 
Nuclear Instruments and Methods in Physics Research Section A, 786, 120-126. http://dx.doi.org/10.1016/j.nima.2015.03.059

[5] Kolata, J.J., Auble, R. and Galonsky, A. (1967) Excitation Energy of the First Excited State of ${ }^{12}$ C, and Observation of a Coherent Doppler Effect. Physical Review Letters, 162, 957-962. http://dx.doi.org/10.1103/PhysRev.162.957

[6] Kiener, J., de Sèrville, N. and Tatischef, V. (2001) Shape of the $4.438 \mathrm{MeV} \gamma$-Ray Line of ${ }^{12} \mathrm{C}$ from Proton and $\alpha$-Particle Induced Reactions on ${ }^{12} \mathrm{C}$ and ${ }^{16} \mathrm{O}$. Physical Review C, 64, Article ID: 025803. http://dx.doi.org/10.1103/PhysRevC.64.025803

[7] Koning, A., Hilaire, S. and Duijvestijn, M. (2013) TALYS (Computer Software). Nuclear Research and Consultancy Group, Netherlands; Atomic Energy and Alternative Energies Commission in Bruyères-le-Châtel, France.

[8] Brun, R. and Rademakers, F. (2015) ROOT (Computer Software). CERN, Switzerland. 